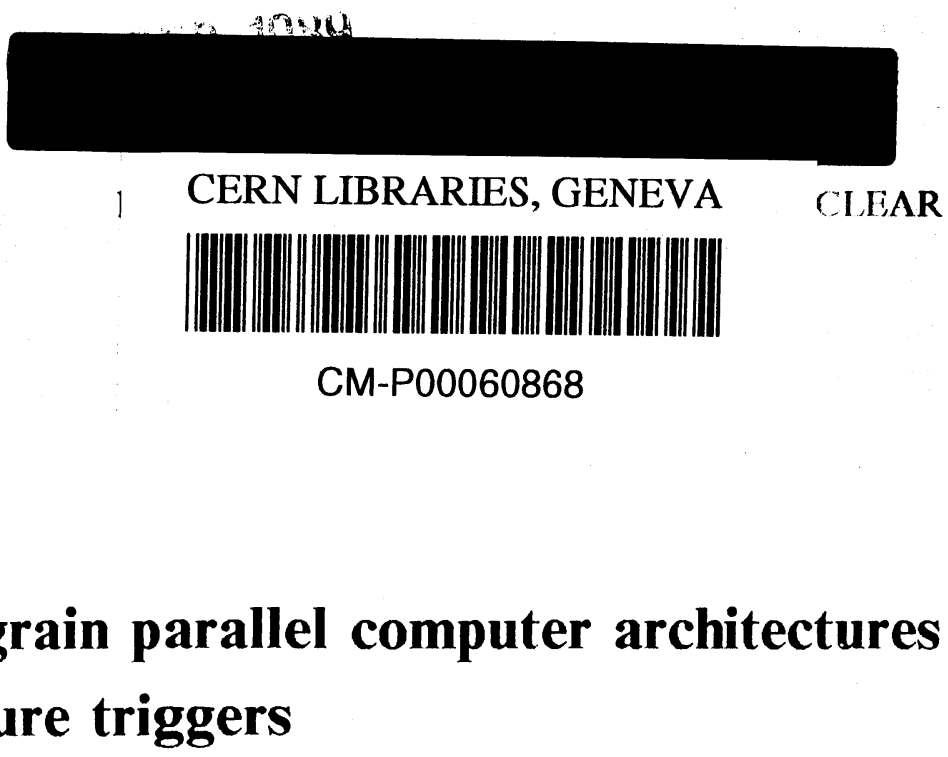

${ }_{2}$

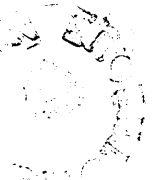

CERN LIBRARIES, GENEVA

CLEAR RESEARCH

CM-P00060868

CERN - EP/89- 116

CERN-LAA RT/89-05

5 September 1989

\title{
Fine-grain parallel computer architectures in future triggers
}

\author{
S.Lone ${ }^{1}$, R.K.Bock,Y.Ermolin ${ }^{2}$, W.Krischer,C.Ljuslin,K.Zografos \\ CERN, Geneva \\ A.Sandoval \\ GSI, Darmstadt
}

\begin{abstract}
As part of the LAA-project at CERN [1], we have studied the suitability of using fine-grain parallel computers in the data-acquisition system of experiments at high energy particle accelerators. Running feature extraction algorithms on such computer architectures may allow to build up triggers or to compact fine-grain data, reducing event rates and/or data volumes.

Our goal was to get a clear understanding of the problems involved in implementing trigger algorithms on parallel structures, and to assess the difficulties and limitations of embedding such structures in data acquisition systems. To this end, we have defined a set of representative benchmarks, and have used them to evaluate several commercially available parallel processor systems, both in hardware implementations and simulation. Overviews of the benchmark and of the architectures, and the numerical results of the benchmark are presented.

In conclusion, the parallel systems studied are seen to outperform other forms of commercially available computers by large factors, for suitable algorithms on partial detector data. They will be serious competitors to custom-designed processors in triggering and data compaction tasks, in future experiments having to deal with high-intensity beams. The applications will be found in clustering, track finding, calorimetry, and in particle identification devices like TRD-s or RICH counters.
\end{abstract}

(Submitted to Nucl.Inst.and Methods in Phys.Research)

\footnotetext{
${ }^{1}$ On leave from SINTEF, Trondheim, Norway

${ }^{2}$ On leave from ITEP, Protvino, USSR
} 


\section{Introduction.}

Studies done for projected future hadron accelerators and their detectors indicate event rates of up to $10^{8}$ events/second, and data volumes greater than 1 Mbyte/event [2]. The high event rate is dictated by luminosity and total cross section, and is needed to study the low-cross section processes of interest. The data volume, assumed larger than in today's detectors by only a moderate factor, results from the desired high resolution of the different detector parts.

If we assume that we want to make use of the fine grained information at the triggering level, programmable and flexible devices with massive compute power will be required. At the expected high data rate, the fastest decisions will have to be taken by analog electronics and fully customized logic; we arbitrarily assume that for a decision in a 'second level' trigger, of the order of 10 microseconds will be available. Fully programmable serial processors are unlikely to deliver the necessary computing capacity alone, although, theoretically, making many of them work in parallel can solve the problem. The more locally an algorithm runs, the more pressing will be demands on short latency, because large buffers are needed to retain data, and severe synchronization problems arise.

In general, the parallel execution of an algorithm on event data may be obtained in various ways; they differ mainly in the way the processors share data by communication; for a detailed discussion, see [3]. All solutions can give high throughput, in number of events treated per second; the difference is mainly the latency: the time that elapses between data first entering processors and the appearance of the result for that data (e.g. an image as defined for our benchmark).

The simplest conceptual way to obtain parallelism is to treat several events separately, with one processor per event and no communication between them. Processors used in this event-parallel way can be conventional microprocessor-based computers, but also simpler processors can operate in event parallelism, as single-instruction-multiple-data (SIMD) machines. The latency for this event-parallel solution is long, and the burden of parallelizing is left to the overall data acquisition system.

To decrease latency, several processors can cooperate on an event by executing the same algorithm on different parts of the event. The processors might have to communicate data in the border regions. One distinguishes between coarse- and fine-grain architectures. The former is usually based on a few (16 - 256) traditional microprocessors with a communications network between. Each of them can then operate on a fraction of the data. The latter has a large number $(1024-65536)$ of simple processors with a network allowing each of the processors to communicate with neighbors, most frequently near neighbors. Each processor typically has access to few data items. The latency is smaller for this solution because data for only one event is loaded, and several processors work on the same event.

$\Lambda$ nother kind of parallelism is obtained by pipelining. This method can be used at many levels of an architecture; inside most modern mainframe computers, there is a number of pipeline stages in the execution of instructions. In an analogous way, a trigger algorithm can be decomposed into a number of steps, to be executed as operations overlapping in time. The latency in this case is dominated by the pipeline speed, and increases only slightly with the number of stages in the pipeline, and with the time spent in each stage.

As we are concerned about long latencies, we have chosen to concentrate on fine-grain parallel architectures with many simple processors. In addition, a pipelined system has been benchmarked. We used for our benchmark algorithms operating on very partial ('local') data. Event parallelism has not been studied, although it should be understood that it will be a likely complement in future sys- 
tems, both to obtain further speedup and for using off-the-shelf standard devices and their operating systems in higher-level triggering, as suggested in [4].

\section{The benchmarks.}

The benchmarks have been described before [5], and in more detail [6]. Seven different algorithms have been defined, in terms of Fortran code. They are not optimized for execution speed but rather meant to give as precise a definition as possible. The algorithms are relatively simple. They are easily re-coded (and have to be so), in order to take advantage of specific architectural properties. They do not demand floating point capabilities. Data have also been supplied along with the benchmark definition, to facilitate testing of the implementations on actual hardware.

We resume, in the following, the main properties of our benchmark algorithms.

The peakfinder algorithm is an image processing task where the same operation is executed for all the pixels in the image. It finds local peaks in an image by computing the average intensity for groups of neighbors of a given pixel, and comparing them with the pixel's own intensity. The input image is 512 by 512 bytes ( 8 -bit intensities), and the result a list of coordinates $(x, y)$ for pixels where the input image met the criterion of the peak definition.

The generic pattern recognition algorithm matches an input pattern to a tabulated parameterized function. The 'hits' in the binary 64 by 64 input image are translated to intensity values in the parameterized space. As a simple model, we define as output parameter the average of these values. The application we have chosen provides a particle velocity using as input $\mathrm{x}, \mathrm{y}$ pairs simulated to correspond to photon signals in a fast ring imaging Cherenkov device with pad readout. Very similar parameterizations can be used for analyzing other types of detectors.

Calorimeter cluster analysis is represented by two algorithms. The first is a simple form of jet/electron identifier. It finds the pixel with the highest intensity, the centrepixel, and then the sum of the intensities (energies) of the centrepixel's nearest neighbors and the sum of the next nearest neighbors. The ratios between the centrepixel, being above a threshold, and the two sums, allow to distinguish between hadronic jets, electrons, and random pileup. The second algorithm is close to algorithms used currently in many off-line programs. Here, instead of using a fixed neighborhood around the pixel with the highest intensity, a variable-size central region is found. The output is again the sum of centrepixels, the nearest and next nearest neighbors. The input is a 64 by 64 subarea of a 512 by 512 10-bit image, and the output consists of three 16-bit numbers.

The missing energy algorithm calculates for a cylindrical detector, in a plane through the centre perpendicular to the cylinder, the sum of the $x$ and $y$ projections of the transverse energy. The input data consists of a list of $\mathrm{x}, \mathrm{y}$-coordinates, and the output of two 16-bit numbers. In our benchmark, about 1200 coordinate pairs were given on average.

Finally, two trackfinding algorithms are given. The Hough-transform ([7], [8]) maps points in the $\mathrm{x}, \mathrm{y}$-plane into sinusoids in the $\rho, \theta$ (Iough-) space. Collinear points result in curves crossing in a single point. The crossing point's coordinates $\rho, \theta$ define a line segment in the $\mathrm{x}, \mathrm{y}$-plane at a distance $\rho$ from the origin and an angle $\theta$ with the axis. Thus, peaks in the pixel intensity of the Ilough-space (a two-dimensional histogram) identify line segments in the original coordinate space. $\Lambda$ list of $x, y-s$ is the input, and the output is a 128 by 128 histogram. The second trackfinding algorithm is called 'grey 
level histogramming' because angles around an interaction point can be introduced as different intensities (grey levels) in a (two-dimensional) image. This algorithm can be used to find tracks if the track origin is known. Grey level (angle) binning allows to histogram the input image, so that the input is a list of $\mathrm{x}, \mathrm{y}$ coordinates with the vertex as origin, and the output is a histogram of 256 16-bit values. Tracks are then found by analyzing the histogram for peaks. The average length of $x, y$ lists used in benchmarking was between 100 and 150 coordinate pairs.

\section{The architectures studied.}

One pipelined system and two (for some problems three) single-instruction-multiple-data (SIMD) systems were evaluated. The pipelined system has been developed for image processing and offers high ('real-time') speed for a number of typical image processing tasks. As fine-grain detector data resemble images and our algorithms can be translated into traditional image processing 'language', using an image processing system is a natural idea. The SIMD systems are 'old' from the architectural point of view. They have become commercially available only through recent implementations in VLSI technology. The systems we have looked at are bit-serial and have a number of processors in the range 1024 to 16384 .

\section{The MaxVideo system.}

This system is manufactured by Datacube (Peabody, MA, USA). The company has produced special purpose image processing systems since 1978. The MaxVideo system consists of individual VME modules realizing typical image processing functions, and of a means of connecting the modules together. It is left to the user to configure his system according to needs ([9], [10]). Many of the typical image processing applications can execute in (or close to) 'video real time', which corresponds to a standard TV frame rate ( $25 \mathrm{IIz}$ for a $512 * 512$ image). Over the years, about 30 different modules have been developed, the most recent ones offering very high performance in terms of equivalent CPU power. Their module interconnect is called Maxbus; it allows a point-to-point connection from a sender to several receivers. $\Lambda$ receiver can also control whether it wants data from one of two senders. There is one connector at each module reserved for the system clock and the others (seven in the current implementation) carry 8-bit data synchronous with the clock. Some modules have the very important feature called Region-Of-Interest (ROI) processing, where one of the buses carries the necessary ROI-timing. When using ROI, an arbitrary window size (from 1 pixel to $4096 * 4096$ ) can be specified in width and height instead of the 'standard' $512 * 512$ and the processing time is proportional to the number of pixels.

Examples of interesting modules are framebuffers of 1024 by 1024 16-bit, or an 8Mega 8-bit, both with ROI capability, an 8 by 8 convolver with arbitrary coefficients, a 64 by 256 convolver with two different coefficients, a processor board for non-linear transformations in a 3 by 3 neighborhood, pointwise $\Lambda L U$-s, multiplexers with up to $64 \mathrm{~K}$ 16-bit lookup table, a histogramming/feature extractor and a board with a general signal processor.

All modules are controlled by setting up registers from the VME-host which could be any VME-based processor; software is provided, with C-source code, for the Unix-based SUN or for OS9-based 68000 systems. The application software can be developed on a Unix-system and later be recompiled for the OS9-system. The control system includes drivers to run in interrupt driven mode and there is a suite of routines for each board at the level of setting up the correct bit field of a register to execute a certain function. 
The modules and the configuration of the system chosen for the benchmark, was primarily with the peakfinding algorithm in mind. However, we tried to implement all the other benchmarks without doing any interconnection changes. This lead in some cases to unnecessary and time-consuming programming, introducing problems and errors that otherwise could have been avoided. However, this was preferred to changing the configuration for each application.

\section{The NCR GAPP.}

The Geometric Arithmetic Parallel Processor (GAPP) is produced by NCR (Fort Collins, CO, USA). It is essentially a single chip containing 72 processing elements (PE) which are controlled in a SIMD fashion ([11], [12]). They are arranged as a mesh-connected two-dimensional array of $12 * 6$ processors where each processor can communicate with its four neighbors. The processor consists of a 1-bit full adder and a set of multiplexers to feed the two operand registers of the adder. There are 128 bits of RAM for each processor. Independent of the adder there is a communication register which can shift data through the PE or out of the RAM. Hence, I/O can overlap other operations in the processor, with only occasional need for processing resources (cycle stealing) to fetch/store I/O data in RAM. One of the operand registers is also taken out of the processor and logically OR'ed together with the corresponding output from all the other PE-s on the chip. The array is packaged in an 84 pin chip out of which 48 are I/O to the border PE-s. The control consists of 20 bits clocked synchronously to all the PE-s: 7 address lines for the RAM, 13 lines to control the multiplexers of the registers, and 1 clock signal, presently at $10 \mathrm{MHz}$. The chip is cascadable, i.e. it is possible to build up an array of the size needed.

The GAPP chip was commercialized as a 'systolic' array chip, which has caused some confusion. This denomination has been used for a great variety of architectures; the GAPP system offered on the market today is a traditional SIMD system with $48 * 48$ PE-s, expandable to $96 * 96$. The host system is a $\mathrm{PC}$ and $\mathrm{I} / \mathrm{O}$ is via the $\mathrm{A}$ T-bus. Programming is done in a C-like language, but should not be confused with high level language programming. The programming tool offers a high-level-language-like way of constructing control loops, but control of the internal resources is at the low level. A simulator is supplied, but based on a PC, only very small configurations can be simulated. This is, however, often enough to test if an implementation of an algorithm works.

The drawbacks of the GAPP architecture are its inability to disable a PE during the execution of an instruction (lack of activity registers), the small size of the internal RAM, and the rather unusual number of processors on chip $(12 * 6)$. The first forces all the PE-s to unconditionally execute the same operations. While this does not exclude the possibility of obtaining the effect of disabling a PE, it does make programming substantially more cumbersome. Further, the 128 bits of internal RAM are quickly exhausted and make the array difficult to use for certain tasks.

On the other hand, the architectural simplicity is an advantage of the GAPP; this makes it easy to build up a system tailored to the application, i.e. one processor per data item. $A$ controller could in the simplest case consist of a 20-bit wide RAM and a counter, plus some additional logic to start and stop taking the global output into account. 


\section{The ASP.}

The Associative String Processor (ASP) architecture has evolved since the early 1970-s, at Brunel University, UK; it is now marketed by Aspex Ltd (Uxbridge, UK) ([13]). Nithough the idea and principal architecture are not new, cost-effective implementations had to wait for the more recent advent of modern VISI design tools and manufacturing technologies. The ASP SIMD architecture attempts to take advantage of the latest advances in technology, most recently seeking fault-tolerant large-scale implementations using wafer-scale integration. The customer is, in principle, offered a building block in VLSI, and given a high degree of freedom in adapting overall design parameters; the number of processors, processor internal resources, $\mathrm{I} / \mathrm{O}$ and overall system design, all can thus be chosen to fit the needs of the application.

The arithmetic unit of the processor (called APE - associative processing element) is a single-bit full adder. Each processor has an internal RAM of typically 32 to 128 bits. In addition, there is a set of bits - 'activity bits', typically 5 to 8 - to be used as control/status bits in the implementation of algorithms. The processor has a parallel comparator of the same size as the data RAM, and activity registers providing the associative function. Through activity registers, a processor is activated for execution by globally comparing the data on the databus and a masking facility. The latter indicates which bits are to be used in the comparison with the content of the internal RAM, and specifies the activity register. The result of the comparison is also output to a line common to all processors, to allow the controller to find out if any of the processors had a match. All processors are connected to a communication network. The network supports two modes of communication: an asynchronous mode primarily intended for exchange of activating signals, and a synchronous mode where data or activating signals are shifted from one processor left or right, to other processors on the 'string'.

A controller broadcasts data, activity bits and control to all the APE-s simultaneously. The normal sequence of instructions is to activate a number of APE-s according to some criterion; then perform some operation on the data in the activated processors; then again activate APE-s according to some new criterion, operate on data in the active processors, and so on. The programming of the ASP is done in a Modula-2 environment. Libraries exist for the most basic functions and for common image processing operations. If the wanted function does not exist in a library, programs have to be written in a medium-level language in knowledge of the architecural details, i.e. with a high learning threshold. A simulator is provided which can run either on a PC or on a SUN workstation.

The controller also has a scalar processor where operations on floating point numbers or integers can be done faster than in the array if the number of operands is relatively small.

I/O to the APE-s is via the controller's broadcast of data, and filling data into the active APE(-s). This serial mode consists of filling 32-bit data words into and taking results out of one APE in 100 nanoseconds. If this is too slow, a possibility exists to exchange data words in parallel over the network to all $\Lambda \mathrm{PE}$-s simultaneously. This allows a trade-off between the number of processors on the chip and the I/O-buffer size.

The ASP benchmark results [14] refer to two different ASP machines. One is called TRAX1 which is being built for (off-line) analysis of large images (3000 by 2000 pixels), as recorded in the streamer chamber experiment NA35 [15]. Tracks in NA35 are to be measured automatically by applying a sequence of image processing tasks. The TRAX1 has a single string of 16384 processors, based on a $(2.0 \mu \mathrm{m}$ CMOS) chip with 64 processors and a 10)ns cycle time. The second ASP we have considered is based on a design in newer technology (1.2 $\mu \mathrm{m}$ CMOS), giving a cycle time of $50 \mathrm{~ns}$ and with wafer-scale integration, 8192 APE-s per wafer. We have assumed this system architecture to be custom-tailored to each of the benchmark algorithms, including its $\mathrm{I} / \mathrm{O}$. 


\section{The AMT DAP.}

The AMT, Reading, UK, Distributed Array Processor (DAP) is also a SIMD architecture whose origin is in the early 1970's ([16]). Developed originally at ICL, successful commercialization again had to wait until recently when the basic processing element was implemented in VLSI. It is a 32 by 32 (or 64 by 64) mesh-connected machine where each processor can communicate with its four neighbors. The processor is bit-serial, has an activity-bit controlling whether the processor writes to its memory or not, and a register intended for high-speed $\mathrm{I} / \mathrm{O}$. Each processor has $32 \mathrm{~K}$ bits of memory or more. The array controller does the instruction broadcast and can load data row- or columnwise to the array. The controller also contains a scalar processor and the processor array is often seen as an extension to the controller's ALU.

The advantage of the DAP is that it is programmable in a high level language (Fortran with extensions for array-structures). This gives a low threshold for a beginner to use the machine. We have, however, observed that Fortran coding results in a performance degradation compared to optimal coding, by a factor of four to ten.

The large per-processor memory of the DAP can also be an advantage, e.g. one or more full images can be stored in the memory at a time. However, this does not help substantially when high throughput and low latency are demanded. Our study objectives did not allow for event parallelism, i.e. for the possibility of entering 1000 events into the system, one for each processor, before starting program execution. A further drawback of the DAP for our study was the rigid packaging into a fixed-size array.

The initial benchmarking done shows that the DAP has no outstanding characteristics of its own compared to the other SIMD-machines, which would justify including this architecture separately. A full benchmark has therefore not been carried out for this architecture.

\section{Benchmark results.}

Implementing an algorithm optimally on a specific fine-grain architecture demands good knowledge of the architecture. The benchmark has therefore been run at three different places according to previous knowledge or field of interest: for the ASP naturally at Brunel University/Aspex, UK, for the GAPP at Iaben, Milano, Italy and for the MaxVideo system at CERN. The intention was to run the benchmark on hardware for all the architectures to get hands-on experience besides confidence in the results. However, only the MaxVideo system has been fully available for benchmarking, the other devices are still in a build-up phase, at time of writing. The results presented for the GAPP and ASP have been obtained using proprietary simulation tools. As processor timing is an integrated part of the simulator, the timing of the computational part can be looked at with confidence.

We have made no clear statements as to how $\mathrm{I} / \mathrm{O}$ should be included in the benchmark, but have indicated that the input of data to processors needs serious discussion as a likely bottleneck. Demanding high compute power and low latency, for large amounts of data, obviously requires high $\mathrm{I} / \mathrm{O}$-bandwidth. In some architectures the $\mathrm{I} / \mathrm{O}$ is embedded and thus naturally limits the combined $\mathrm{I} / \mathrm{O}$-compute bandwidth. In other architectures, a certain $\mathrm{I} / \mathrm{O}$ implementation is built in to meet anticipated needs, but the architecture leaves room for increasing the $\mathrm{I} / \mathrm{O}$ capacity. Including $\mathrm{I} / \mathrm{O}$ fully in the benchmark would require specific studies and, in particular, specific assumptions about the other components of the surrounding data acquisition system, starting with frontend electronics. We have 
therefore explored, in our benchmarking, not only the algorithm and $\mathrm{I} / \mathrm{O}$ performance of a specific implementation of the architecture, but also the limits that we would have to expect if we wanted to achieve optimal matching of architectural concept and algorithm.

\section{Results of the peakfinder.}

The peakfinder is simple in terms of coding and is a typical low-level image processing task. The same operation is to be executed on all the pixels and should therefore adapt well to parallel architectures. The performance of the VAX has been added to put the results in perspective. No optimization of the code was done, the Fortran code was compiled as given and run. The timing is given excluding the loading of data. The results of the peakfinding algorithm are tabulated in Table 1.

\begin{tabular}{|c|c|c|}
\hline Architecture & Execution Time & Comment \\
\hline VAX 8300 & seconds & Performance $1-2$ VAX $11 / 780$ \\
\hline MaxVideo & millisec & Present config., pipeline $10 \mathrm{MHz}$ \\
\hline GAPP & $\begin{array}{l}28.1 \text { millisec } \\
47.5 \text { microsec }\end{array}$ & $\begin{array}{l}48 * 48 \mathrm{PE}-\mathrm{s}, \mathrm{I} / 010 \mathrm{Mb} / \mathrm{s} \\
512 * 512 \mathrm{PE}-\mathrm{s}, \mathrm{I} / 0 \text { matched }\end{array}$ \\
\hline ASP & $\begin{array}{l}1.7 \text { millisec } \\
23.4 \text { microsec }\end{array}$ & $\begin{array}{l}\text { TRAX1, i.e. } 16 \mathrm{~K} \text { processors } \\
127 * 4 \mathrm{~K} \text { processors }\end{array}$ \\
\hline DAP & $\begin{array}{ll}46 & \text { millisec } \\
13 & \text { millisec }\end{array}$ & $\begin{array}{l}32 * 32 \text { PE }-\mathrm{s}, \text { Fortran } \\
\text { idem, Apal (machine language) }\end{array}$ \\
\hline
\end{tabular}

Our MaxVideo configuration was tailored to this algorithm and the performance is the number of pixels times the cycle time (i.e. 100 nanosec), plus an overhead per line. This is the basic limitation of the pipeline. $\Lambda \mathrm{n}$ increase in pipeline speed would directly scale up the performance. One could also imagine dividing the total image into 2 or 4 parts, with a separate pipeline for each part; this would provide a corresponding speedup factor. However, this split would result in bordering problems for many operations; we have not attempted to pursue the idea.

In the GAPP a PE is assigned to each pixel. It uses all the 128 bits of memory during the calculation. 'The first number given in the table is for the $48 * 48$ system; new data is assumed loaded and exchanged with results in time overlap with calculation. This figure is limited by the $\mathrm{I} / \mathrm{O}$ to the array which consists of one row (48) of 8 bits parallel shifted into the $\mathrm{I} / \mathrm{O}$-buffer and arranged into bit-vertical entities that can be loaded into the array (so-called 'corner turning'). The 47.5 microseconds is the time it takes for executing the algorithm in the Main Array. Provided there is one PE/pixel and that the $\mathrm{I} / \mathrm{O}$ can be matched - i.e. smaller than the $47.5 \mu$ s and overlapped with the computation - this is the lower limit for the architecture and algorithm. 
The ASP TRAX1 assigns again one APE per pixel, and can thus treat a patch of $32 * 512$ pixels at a time. 19 of these patches have to be dealt with serially. The optimal implementation needs a configuration of 1274096 string-APE-s (=520192). Each patch is then $8 * 512$ and 4 pixels are calculated in each. The $\mathrm{I} / \mathrm{O}$ time has to be added as it is not overlapping computation. The theoretical lowest possible would be $400 \mathrm{~ns}$ ( $50 \mathrm{~ns} * 8$ bits).

The first DAP figure corresponds to the performance reached when Fortran is used to implement the algorithm, while the second is obtained with the use of assembler language. Converting the data to a format suitable for the bit-serial machine is assumed done outside the DAP-array. Normally, when data is loaded via the host, the 'turning' from word-parallel to bit-serial is done in the DAP itself. Data formatting can be done in an external module, entering data via the $\mathrm{D}$-plane at $50 \mathrm{Mb} / \mathrm{sec}$.

\section{Results of the remaining algorithms.}

The results of the remaining algorithms are presented in Table 2. Again, to put the results in perspective, VAX figures are included.

Table 2: Execution time for the remaining algorithms

$\begin{array}{lcccc}\text { Algorithm } & \begin{array}{c}\text { MaxVideo } \\ {[\mu \mathrm{s}]}\end{array} & \begin{array}{c}\text { GAPP } \\ {[\mu \mathrm{s}]}\end{array} & \begin{array}{c}\text { ASP } \\ {[\mu \mathrm{s}]}\end{array} & \begin{array}{c}\text { VAX } 8300 \\ {[\mathrm{~ms}]}\end{array} \\ \begin{array}{lccc}\text { Pattern } \\ \text { recognition }\end{array} & 1000 & 725 & 114 & 13.5 \\ \begin{array}{l}\text { Calorimeter, fixed } \\ \text { neighborhood }\end{array} & 615 & 384 & 50.6 & \\ \text { Calorimeter, variable } & - & 725 & 119 & 22.5 \\ \text { neighborhood } & & 780 & 278 & 630 \\ \text { Missing energy } & - & 8000 & 150 & \\ & & & 260 & \approx 20 \\ \text { Hough-transform } & 838 & 17500 & 1900 & \\ & & 136 & 956 & 500 \\ \text { Grey level } & 16 & 4000 & 190 & 3.1 \\ \text { histogram } & & & 16.8 & \end{array}$




\section{The pattern recognition implementations.}

The MaxVideo system performs the logical AND using a lookup table, and then makes use of the large area convolver to add up the $64 * 64$ numbers. The actual MaxVideo configuration makes it necessary to send out data twice as there is only one module capable of large area convolution. This gives the first timing. However, with only one additional standard module the improved performance figure would be obtained.

The GAPP-figures assume a $64 * 64$ PE-configuration, and the first number is with the present I/O. The second number is the computational part of the algorithm - a logical AND, a 1-bit and an 8-bit global add - and represents thus the performance if the $\mathrm{I} / \mathrm{O}$-requirements are matched.

The ASP solution makes use of the asynchronous communication to count the number of non-zero pixels and to add up the corresponding values in the parameter space. The optimal solution has 4096 APE-s and in the TRAX1 case only 4096 of the 16384 APE-s are used. There is also a difference in the way scalar temporary results are treated which gives a factor between the two implementations different from two (which is the assumed performance increase for the new chip design).

\section{Calorimeter, fixed neighborhood}

To find the maximum intensity pixel with our present MaxVideo modules is not straightforward. The solution chosen, however, does the convolutional part of the algorithm for all pixels, but lets through only those being 'real' peaks and zeros otherwise. Then the feature extraction module picks out those data being above a certain threshold, giving the host a small array to scan for the highest intensity pixel and the neighborhood sums. The timing is again the time to send a 64 by 64 pixel window through the pipeline (plus the overhead per line). It should be noted that the input data are 10 bits, but the convolution modules at present work with only 8 bit inputs.

The GAPP results assume a $64 * 64$ array and the first number is dominated by the $\mathrm{I} / \mathrm{O}$-time. The second number is the main-array time to mark the highest pixel value and calculate the two neighborhood averages and defines thus the lower limit assuming I/O can be tailored.

The ASP marks the highest intensity pixel, calculates the two neighborhood averages and then assumes the remaining two additions and divisions to be done in the scalar processor. The lower number for the optimized implementation needs a 4096 APE ASP.

\section{Calorimeter, variable neighborhood}

In our actual MaxVideo configuration the data would have to be recirculated to expand the centre pixel area, as the process is iterative. For performing the operation in a single pass, a large number of identical boards in the pipeline could be considered; we have not followed this idea seriously, and have therefore not implemented this algorithm.

The GAPP figure is the timing to find the maximum intensity pixel, execute the 'worst case' expansion of the centre-region, and then find the necessary sums.

'The ASP expands the central region first horizontally by asynchronous communication, then vertically by synchronous communication, until no further expansion can take place. The optimal solution needs 4096 APE-s and 100 scalar operations partially overlapped with the parallel processing. The 
timing is given for a window with a few cycles of expansion of the centre pixels. The worst case expansion would lead to an increase of the timing with only $87 \mu$ s to a total of $237 \mu$ s compared to the $150 \mu$ s.

\section{Missing energy}

The MaxVideo system, by its bus width and register size, does not allow a solution with the required precision. Products can be formed between two 8-bit inputs with 16-bit output, but the convolver that could be used for accumulation of the intermediate results, works with only 8-bit inputs. We have therefore not included this algorithm in the results.

The GAPP figure is a 'qualified guess', which has not been properly simulated. The memory size of the processing elements is too small to implement an efficient solution.

In the ASP each APE gets a $\sin (\theta) * \sin (\phi)$ and $\sin (\theta) * \cos (\phi)$ loaded into the data register together with the corresponding energy. Then the $\mathrm{x}$-component for all the $\Lambda \mathrm{PE}$-s is calculated and those different from zero accumulated. The same is done for the y-component. The worst case timing would be $7.2 \mathrm{~ms}$ for TRAX1 while the optimal solution would need $248 \mu$ s using 262144 APE-s. However, the benchmark data had in average 1000 data items and thus a 8192 string is sufficient giving the timing $130 \mu \mathrm{s}$. In the latter case it should also be noted that the data would have to be loaded through a lookup table.

Overall, this algorithm seems least suited for the architectures studied, despite its simplicity. Two facts contribute to this: the only common parallel operation is a multiplication, but both factors are pixel-dependent; and the final global summing is a notorious non-SIMD operation.

\section{Hough-transform}

The Hough-transform was implemented by our group as a special hardware module in the MaxVideo system. The $\mathrm{x}, \mathrm{y}$ coordinates are loaded over the VME-bus at present; in a future implementation, using a feature-extraction module able to supply data via the Maxbus, the Hough-transform board will be accelerated using this (faster) bus. The current version updates the Hough-array once per $50 \mathrm{~ns}$, per point and angular bin. Various speedup possibilities by parallelizing exist, and have been discussed in [8].

In the $G \Lambda P P$, the $x, y-s$ are loaded and then 128 cycles executed in which the bin-numbers are calculated according to $x, y$. For each cycle an exchange of bin-numbers and a new set of cosine(angle) sinus(angle) is executed overlapped with computation. The first number indicates the time taken when up to $48 * 48(=2304) \mathrm{x}, \mathrm{y}-\mathrm{s}$ can be processed at a time for an angle. The histogramming is not done in the G $\Lambda P P$-array, but in an external scalar processor. In the actual system the scalar processor is the host and it updates the histogram in parallel with the next set of bin-numbers being calculated in the GAPP-array. The first number indicates the time taken when up to $2304 \mathrm{x}, \mathrm{y}-\mathrm{s}$ can be processed at a time for an angle. How many updates of the histogram the host can update in the corresponding time, has not been examined. The second number is the time to calculate the bin-numbers for one angle, for all $\mathrm{x}, \mathrm{y}-\mathrm{s}$ simultaneously; it is thus the theoretical lower limit provided enough hardware is available to calculate all the angles in parallel, e.g. $128 * 48 * 48$ if $2300 \mathrm{x}, \mathrm{y}-\mathrm{s}$ is an expected upper limit. The time to update the histogram must be added.

The two ASP implementations have a factor two in difference due to the doubling of clock speed assumed for the new chip design and the optimal solution being identical to the TR $\triangle X 1$. Each APE 
has a different $\rho, \sin (\theta)$ and $\cos (\theta)$ according to the position of the APE in the Hough-array. The $\mathrm{x}, \mathrm{y}-\mathrm{s}$ are broadcast to the ASP string and the APE-s calculate a $\rho$. If the calculated $\rho$ in an APE correspond to its position in the array, the Hough-array counter is incremented. In $7.3 \mu$ s the two-dimensional histogram is updated for one $\mathrm{x}, \mathrm{y}$-pair and 128 angles.

\section{Grey level histogram}

The grey level histogram is implemented as a table lookup in the MaxVideo system, which produces the bin-number to histogram. The hardware is used in an optimal way, storing coordinates as pixel intensities in two separate ROI stores. As we had no access to the (now available) ROI-compatible version of the histogramming module, we give our results without this function; the timing with histogramming included would be the same with the module available, and is only a function of the number of $\mathrm{x}, \mathrm{y}$-pairs to histogram.

The figure given for the GAPP-array is for an assumed $256 * 256$-configuration, it is dominated by the I/O-time. In fact, the only part of the algorithm done in the array is the simple AND of the input image with the bin-number mask, which takes $2.6 \mu \mathrm{s}$. Histogramming is not included, and is thought a host task.

The ASP solution is most efficient when pixels belonging to a bin are loaded in consecutive APE-s belonging to an ASP substring. Then the histogram is obtained by counting within each substring, thus constructing all bins simultaneously. The TRAX1 solution has to deal with the problem in patches executed serially, while the optimal solution needs a number of APE-s equal to the number of bins, times number of pixels per bin $(255 * 320=81600)$.

\section{Evaluation of the architectures.}

The MaxVideo system has its $\mathrm{I} / \mathrm{O}$ embedded in the architecture, and the combined bandwidth for computation and $\mathrm{I} / \mathrm{O}$ is $100 \mathrm{~ns}$ per input value. The modules available make it comparatively easy to implement typical low level image processing tasks. It is extendable in terms of adding functions in the pipeline, and one can execute independent tasks of an algorithm in parallel by implementing separate pipelines. However, an algorithm must do with the resources given, e.g. in terms of bit resolution or input bits for a lookup table. Program debugging can also be difficult, possibly due to lack of a simulator. Once registers are loaded and a data transfer started, the possibilities to find out where in the chain an error occurs, are limited (note that efficient programming of SIMD) systems is an equally time consuming task). Iowever, the system is available and the Maxbus interconnection strategy seems to have become an industry standard with support and modules from several independent manufacturers. The performance figures show that it could be used for triggering purposes even for large 'images', if the performance needed is in the millisecond range. Suitable algorithms for small image or list size can execute surprisingly fast. If technology evolution holds its promises for the next years, one might see a similar system with ten times the performance over the present system (i.e. with a pipeline speed of 100 MHz).

The GAPP system has been widely referred to in the literature, but it turned out difficult to get the hardware delivered and to get support from the manufacturer. The way the component was originally introduced - as a systolic building block - has not been extensively exploited with respect to the benchmark. Some of the results might have been better with further studies of a more appropriate 
matching of architecture and algorithm. In most of the benchmark algorithms the implemented $\mathrm{I} / \mathrm{O}$ is the limiting factor, but relatively simple VLSI interfaces would alleviate the problem.

The ASP seems to give very good overall results on our benchmark. However, for the highest-performance results we should remember that they are extrapolated simulations, this architecture still is in the phase of technology exploration. Until at least prototypes have been demonstrated, the feasibility of building a system with 1/4 million APE-s and matching I/O remains a (justified) hope. Over the next few years, wafer-scale integration combined with $\mathrm{I} / \mathrm{O}$ hybrids on a substrate can be expected to exist, success being mostly a question of resources. On the other hand, it should be mentioned that the architecture is rather unconventional from a programming point of view. It has a higher learning threshold than the more conventional ones, and it is time consuming to understand how to use the internal resources to implement a function optimally. Although a high level language environment is provided with libraries for the basic needs, optimal utilization for a given task not existing as a library function has to be programmed at the low level.

\section{Evaluation of the results.}

The performance factors between MaxVideo and VAX, and between the optimistic ASP-figure and VAX, are tabulated in Table 3.

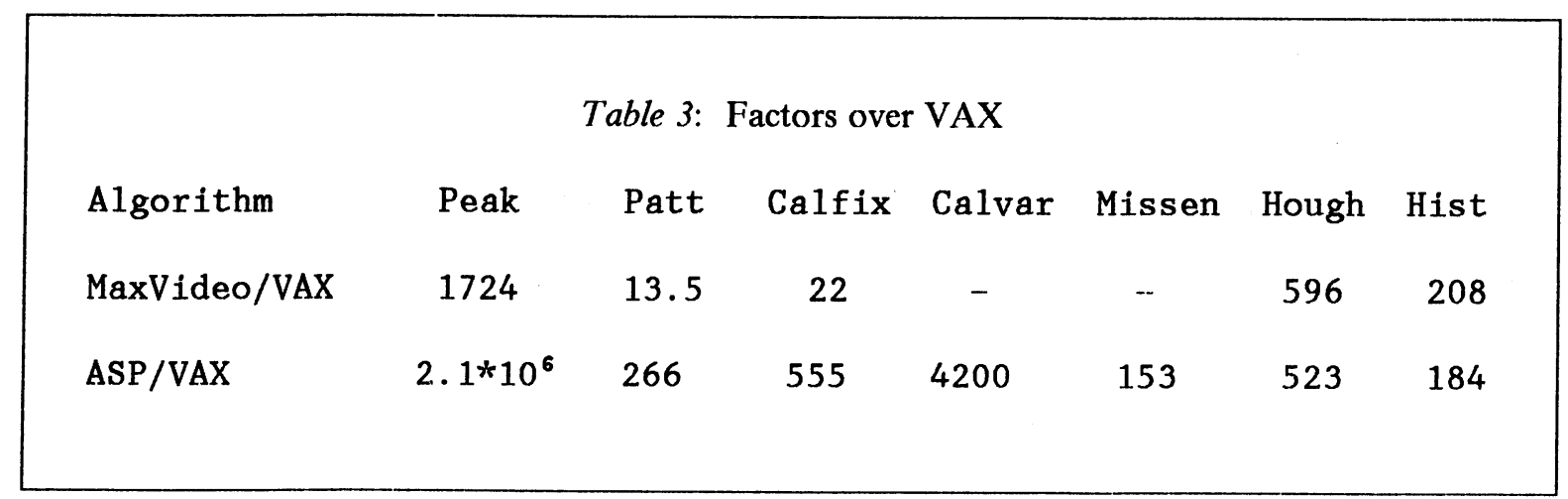

The numbers should not be taken literally, with the indicated precision, and it should be kept in mind that the VAX figures are from Fortran code with not much effort in optimization of the code. On the other hand, the ASP-figures give mostly worst-case times, where the input data were specified as lists, whereas the VAX timing is for the actual data given with the algorithm.

Available modern serial systems based on RISC processors perform at a level of about $10 \mathrm{VAX}$ equivalents (Mips). Comparing these against the MaxVideo, we have to change numbers in the table by a factor of ten, hence the latter performs better by a factor from 1 to 170 , depending on the parallelism in the problem. Likewise, comparing RISC processors available in $2-4$ years (predictions say 100 Mips) with the ASP system available on the same time scale, the performance ratio is from 2 to $210^{4}$, in favour of the ASP. 


\section{Conclusions}

Our benchmark was set up to explore how today's commercial parallel computer systems would perform when used for 'intelligent' low level feature extraction in detector components on future high-luminosity accelerators. Our results show that the necessary compute power is within reach, if a special effort is devoted to packaging and interfacing, most likely at the VLSI design level. For certain tasks, the fine-grain parallel architectures are unequalled and far superior to general-purpose serial computers.

For other types of algorithms, working on previously extracted features represented by lists of $\mathrm{x}, \mathrm{y}-\mathrm{s}$, fast serial processors seem more competitive, if we think of RISC-s of $\geq 100 \mathrm{Mips}$ performance, expected to exist in a few years time. The time for such algorithms, typically applied after thresholding ('zero suppression'), scales with the actual number of data items, i.e. varies from event to event. Fine-grain parallel computers treat a fixed number of items simultaneously, thus giving a 'worst case' timing for such dynamic problems. A successful combination of the two architectures seems to be the natural answer, the mix depending strongly on precise detector and trigger parameters.

There exists a mismatch between compute power and $\mathrm{I} / \mathrm{O}$, if pixel arrays are assumed large. An image of 512 by 512 bytes image requiring analysis every $10 \mu$ s translates into an I/O-rate of $26 \mathrm{~Gb} / \mathrm{s}$; a 64 by 64 array translates into $410 \mathrm{Mb} / \mathrm{s}$. For the first figure, extensive parallelism will be needed, while the latter rate is not beyond present performances by orders of magnitude; the $\mathrm{I} / \mathrm{O}$-rate for the described next generation ASP system is $640 \mathrm{Mb} / \mathrm{s}$ per module.

In order to assess realistically the possibility for embedding such systems in an experiment's data stream, more detailed information on other system parts is required. With parallel and pipelined architectures not only available off the shelf in fully packaged form, but also as libraries for the VLSI designer, our results should encourage experimenters to include systems of the kind we have studied in their planning and designing of future data acquisition and trigger systems, as a serious alternative to custom-designed processors.

Our benchmark algorithms are characterized by using local data, and results are strongest where border problems can be avoided. On feeding data from the detector readout into many processors working in parallel, this may translate into data duplication for overlap regions. Applications of the algorithms can be found in various detector parts: They are part of low-level clustering in one- or two-dimensional chambers, they are needed for track finding, electron/hadron identification, jet/pileup separation, and will also be useful in particle identification devices like transition radiation detectors or ring imaging Cherenkov counters (e.g. with pad readout).

\section{Acknowledgements}

We want to express our gratitude to the LAA project leader, Prof. A.Zichichi, for his continued support. We also thank Drs. J.Dhatt and A.Krikelis from Aspex/Brunel, who have run the benchmark on the ASP, and Prof.M.I.ea (Brunel) who has been crucial in generating and maintaining the high-energy physics interest of $\Lambda$ spex. Equally, Drs. M.Caenazzo and E.Macavero from Laben (Milano) must be thanked for running the GAPP simulation, and Dr. S.Reddaway from AMT, for help concerning the DAP. 


\section{Bibliography}

1. A. Zichichi : The LAA Project, CERN, 25 June 1987.

2. Physics at the Superconducting Supercollider, Proceedings of the 1986 Snowmass Summer Study, R.Donaldson and J. Marx, Editors.

G.Brianti: The Large Hadron Collider in the LEP tunnel, Workshop on Physics at Future Accelerators, La Thuile/Geneva, January 1987, CERN 87-07.

Experiments, Detectors, and Experimental Areas for the Supercollider, Workshop Proceedings Berkeley 1987, R.Donaldson and M.G.D.Gilchriese, Editors.

M.G.D.Gilchriese: Triggering Requirements for SSC Physics, Proceedings of the Workshop on Triggering and Data Acquisition for Experiments at the Supercollider (January 1989), R.Donaldson, Editor, SSC-SR-1039, April 1989.

3. R.W.Hockney, J.R.Jesshope: Parallel Computers, Adam Hilger Ltd., Bristol 1981.

L.Uhr, K.Preston, S.I.evialdi, M.J.B.Duff (eds.): Evaluation of Multiprocessors for Image Processing, Academic Press 1986.

S.Y.Kung: VLSI Array Processors, Prentice Hall 1988.

4. I.Gaines: Microprocessors and other Processors for Triggering and Filtering at the SSC, Proceedings of the Workshop on Triggering and Data Acquisition for Experiments at the Supercollider (January 1989), R.Donaldson, Editor, SSC-SR-1039, April 1989.

5. R.K.Bock et. al : Feature Extraction in Future Detectors, 4-th Pisa Meeting on Advanced Detectors, May 1989, Proceedings to be published in Nucl.Inst.and Methods in Phys.Research.

6. R.K.Bock, W.Krischer, S.Lone : Benchmarking computer architectures for HEP real-time algorithms, CERN - LAA/RT/88-08 (internal report).

7. R.O.Duda and P.E.Hart: Use of the Hough Transformation to Detect Lines and Curves in Pictures, Comm. ACM Vol 15 No 1, 1972.

8. Y. Ermolin, C. Ljuslin : The Use of Image Processing in Tracking, 4-th Pisa Meeting on Advanced Detectors, May 1989, Proceedings to be published in Nucl.Inst.and Methods in Phys.Research.

9. R.J.Berger, B.Unger : Modular Real-Time Image Processing Hardware as a Means to Offload Computationally Intensive Tasks in Artificial Vision, SPIE vol. 635, Applications of Artificial Intelligence III (1986).

10. DATACUBE : Maxvideo User Manuals.

11. R.Davis et al. : Series of 5 articles on systolic arrays, Electronic Design, Oct 31, 1984 - Jan $10,1985$. 
12. NCR GAPP Application Notes : NCR 45CG72, NCR Corp 1985.

13. R.M.Lea: The ASP: a cost-effective parallel microcomputer, IEEE Micro, Volume 8, No.5, October 1988.

14. J.Dhatt et al.: Performance of the ASP on the LAA Benchmark, Document Number 501-001, Aspex Microsystems, June 1985, (also CERN - LAA/RT/89-08 (internal report)).

15. NA35 Experiment, Athens, Bari et al.: Study of Relativistic Nucleus-Nucleus Collisions, CERN-PSCC/82-1/P53, CERN-SPSC/85-26/M404 etc.

16. DAP 500 Manuals: Active Memory Technology Ltd, Reading, UK, 1987. 